\title{
SITUACIÓN ECONÓMICA DE LA PRODUCCIÓN DE BOVINOS DE CARNE EN EL ESTADO DE CHIHUAHUA, MÉXICO ${ }^{1}$
}

\author{
Nicolás Callejas-Juárez ${ }^{2}$, Heriberto Aranda-Gutiérrez², Samuel Rebollar-Rebollar ${ }^{3}$, \\ Martha Leticia de la Fuente-Martinez ${ }^{2}$
}

\section{RESUMEN}

Situación económica de la producción de bovinos de carne en el estado de Chihuahua, México. El objetivo del presente estudio fue analizar la situación microeconómica de Unidades Representativas de Producción (URP) del sistema de producción vaca-becerro en el estado de Chihuahua, México. Se analizaron cuatro escalas de producción (20, 40, 200 y 500 vientres en edad productiva) en cuatro regiones del estado de Chihuahua, México. Con la metodología de panel se obtuvieron costos e ingresos en 2009, con la participación de cinco a diez productores por panel. Se utilizó el modelo de simulación económica MEXSIM de la Universidad de Texas A\&M, adaptado para México por la Red Nacional de Investigación en Política Pública. La viabilidad económica medida a través de la probabilidad de obtener reservas finales de efectivo negativas y perder capital, fue buena para CHBC20, CHBC200 y CHBC500 y moderada para CHBC40. La utilidad neta más baja por vientre fue para CHBC40 (\$27,76 dólares) y la más alta para CHBC20 (\$ 403,42 dólares). La relación beneficio-costo fue de 2,16 y 1,78 para CHBC500 y CHBC200, respectivamente. Todas las URP obtuvieron el ingreso neto suficiente para cubrir sus gastos familiares, excepto CHBC20 que generó 70\%. Las URP con mayor escala fueron más rentables que las pequeñas y todas trabajaron sobre su punto de equilibrio. El margen de ganancia fue mayor cuanto más grande fue la escala de producción, con lo que se mostró evidencia de economía de escala.

Palabras clave: vaca-becerro, tamaño de hato, carne de res, becerros al destete.

\begin{abstract}
Assesment of economic indicators for cow and calf production in Chihuahua state, México. The objective of this work was to evaluate the microeconomic situation through the use of efficiency indicators, technical, economic and financial for production representative units (PRU) in cow-calf systems. Four scales of production (20, 40, 200 and 500 sows) comprising individuals in reproductive age were analyzed in four regions of the state of Chihuahua, Mexico. By using a panel methodology, costs and revenues were obtained during 2009 with the participation of 5-10 panel producers. We used the MEXSIM economic simulation model of the University of Texas A\&M, which had been previously adapted to Mexico by the National Network of Public Policy Research. It was found that the economic viability, as measured by the probability of negative ending cash reserves and capital loss, is good for CHBC20, CHBC200 and CHBC500 and moderate for $\mathrm{CHBC} 40$. The lowest net income per sow was at $\mathrm{CHBC} 40(\$ 27,76)$ while the highest was for $\mathrm{CHBC} 20$ $(\$ 403,42)$, and the best and worst benefit-cost ratios were 2.16 and 1.78 for CHBC500 and CHBC200, respectively. All PRUs obtained sufficient net income to cover family expenses, except for CHBC20, which only covered $70 \%$ of all expenses. The URP more scale were more profitable than small and all worked on their balance point. In fact profit margins were greater the greater the production scale, which suggests evidence of economies of scale.
\end{abstract}

Keywords: cow-calf systems, herd size, beef cattle, calves at weaning.

\footnotetext{
Recibido: 7 de enero, 2013. Aceptado: 18 de marzo, 2014. Proyecto de investigación "Formación y análisis microeconómico de unidades representativas de producción bovinos carne en México", financiado por la Subsecretaria de Fomento a los Agronegocios de la SAGARPA.

2 Universidad Autónoma de Chihuahua. Facultad de Zootecnia y Ecología. Chihuahua, Chih., México. Periférico Francisco R. Almada Km 1. CP 31031.ncallejas@uach.mx (autor para correspondencia), heryaranda@gmail.com,mfuente@uach.mx

3 Centro Universitario Temascaltepec-Universidad Autónoma del Estado de México (UAEM), México. Barrio de Santiago s/n, Temascaltepec, CP 51300.samrere@hotmail.com
} 


\section{INTRODUCCIÓN}

En el siglo XXI, la actividad agropecuaria es un instrumento fundamental para el desarrollo sostenible y reducción de la pobreza. Tres de cada cuatro personas pobres en países en desarrollo viven en zonas rurales, de los cuales 2100 millones subsisten con menos de dos dólares/día y 880 millones con menos de un dólar (Banco Mundial, 2007).

En 2009, el ganado representó el 40\% de la producción mundial y contribuyó a la subsistencia y seguridad alimentaria de casi mil millones de personas, aporta $15 \%$ de la energía alimentaria mundial y $25 \%$ de las proteínas de la dieta que utiliza; además, $60 \%$ de los hogares rurales tienen ganado bovino (FAO, 2009). La FAO (2013) reportó que la producción mundial de carne en 2011 fue de 593 millones de t, mientras que en 2000 de 458 millones de t: $27,7 \%$ de cerdo, 17,2\% de pollo, $11,6 \%$ bovino, $1,7 \%$ ovino, $1,2 \%$ caprino y el resto otras. Asia aportó 41,9\%, América 31,6\%.

El volumen mundial producido fue de $62,8 \mathrm{mi}-$ llones de t de carne de bovino, $12 \%$ más que en 2010. El continente americano aportó 49\%, Europa 21\%, Asia $18 \%$ y África $7 \%$. Los países más importantes fueron EUA 19,1\%, Brasil 14,4\% y China 9,8\%; así mismo, México ocupó el séptimo lugar con 2,9\%. El aumento en el volumen producido de carne se debió a la adopción de tecnologías que incrementaron la productividad, mejorando la cría, alimentación, control de enfermedades, comercialización y el transporte (FAO, 2009). De 1987 a 2007 el aumento en la producción de carne bovina fue inferior al de las otras especies: bovino $21,6 \%$, cerdo $81,6 \%$ y aves $141,8 \%$.

Al considerar la ganadería bovina, independientemente de las demás especies, se concluye que los ganaderos pequeños no están en niveles de extrema pobreza, pero sí tienen restricciones para su desarrollo. Los problemas más importantes del Sector Agropecuario de México son: escaso o nulo financiamiento, sequías, capacitación, tecnificación, sanidad animal y condiciones desventajosas de comercialización de productos cárnicos (Osuna, 2003). Estudios de caso realizados por Delgado et al. (2008) mostraron que los productores pecuarios comerciales en pequeña escala en Tailandia, Brasil, India y Filipinas pueden ser competitivos, incluso en un sector en rápida evolución, pero deben contar con el apoyo institucional adecuado y reducir el costo de oportunidad de su mano de obra.
El uso de suplementos en bovinos productores de carne, en el norte de México, ha crecido debido a la baja producción natural de forraje, que sumado al escaso aumento en el precio de venta de la carne, incrementa el costo de oportunidad de inversiones. Aksoy et al. (2009) encontraron que en la producción de carne bovina en Turquía, el costo del concentrado y heno afecta significativamente el ingreso del productor, y en la producción de leche, el costo del concentrado y la mano de obra eventual hacen menos viable su producción.

La producción de bovinos de carne es de gran importancia en el contexto socioeconómico de México, ya que proporcionan alimentos, materias primas, divisas y empleos; pues se realiza sin excepción en todas las regiones ecológicas del país (Tinoco et al., 2011).

La ganadería bovina en México se desarrolló durante décadas, a través de un modelo extensivo con un fuerte impacto ecológico. Su crecimiento y rentabilidad se fundaron en la extensión de la superficie de pastoreo (Chauvet, 1997). Sin embargo, la continua fragmentación de unidades de producción tiene impactos negativos en el sistema productivo.

La ganadería bovina productora de carne en México se liga al comercio internacional a través de la exportación de becerros para engorda a Estados Unidos de América (EE.UU.), y cortes de carne a Japón y Corea. El ciclo de exportaciones presenta dos etapas: alta, entre septiembre y marzo y baja, de abril a agosto. Según datos del SNIIM (2013) las exportaciones de ganado bovino en pie de México fueron poco más de 1,2 millones de cabezas en 2012 (74\% becerros y $26 \%$ vaquillas).

El sistema de producción vaca-becerro en México tiene como objetivo la producción de becerros al destete y predominante con razas europeas, sin rasgos de ganado lechero; debido a que producen los mejores genotipos para la engorda estabulada, semiestabuldada o en pradera. La producción comienza con la parición a partir del mes de marzo y finaliza ocho meses después con el destete del becerro, con un peso vivo que varía entre 150 y $200 \mathrm{~kg}$. Posteriormente, los becerros son acondicionados (repasto) hasta un peso vivo de 230 a $320 \mathrm{~kg}$, para su exportación o engorda nacional. Las variables escala de producción, edad de la vaca, relación vaca-toro, la alimentación y el manejo determinan la eficiencia técnica y económica de la producción. La ganadería de Chihuahua se caracteriza por la pro- 
ducción de becerros al destete para la exportación, en la cual se requiere de un gran número de hectáreas por unidad animal (por arriba de 20 hectáreas) y presenta una escasa precipitación a lo largo del año (Rodríguez, 2008). En Estados Unidos existían aproximadamente un millón de granjas con bovinos del sistema vacabecerro, que generaron $\$ 40,5$ mil millones en ventas y representaron $21 \%$ del valor total de mercado de los productos agrícolas y el primer lugar en ventas entre todos productos básicos (United States Department of Agriculture, 2007).

La ganadería bovina, en Chihuahua, es propicia bajo el sistema de pastoreo extensivo, que consiste en utilizar grandes extensiones de agostaderos, inversiones en pie de cría, bajos insumos, reducido capital fijo y mínima fuerza de trabajo. La calidad genética del ganado bovino producido en la entidad se reconoce en el ámbito nacional e internacional, la cual se refleja en las más de 350 mil de cabezas promedio exportadas anualmente. La actividad tiene potencial para desarrollarse en una superficie de casi 18 millones de hectáreas de agostaderos (72\% de la superficie estatal), y representa $17,8 \%$ de las actividades agropecuarias, además, dejó una derrama económica en 2009 mayor a los 3411 millones de pesos. En 2011, se produjeron 99829 toneladas de carne en canal de bovinos, que representó el cuarto lugar nacional. Los distritos más importantes fueron Cuauhtémoc $(45,8 \%)$, Casas Grandes $(13,5 \%)$, Parral $(4,3 \%)$ y Chihuahua $(3,1 \%)$. El distrito de parral se caracteriza por la producción de leche y los demás por la exportación de becerros (SIAP, 2013). En ese mismo año, el Gobierno reportó que la ganadería había sido afectada por escasez del agua y pérdidas por muerte de ganado, debido al empobrecimiento de pastizales, causado por un mal manejo de la capacidad de carga y disminución de la cantidad de agua de lluvia. Un impacto importante que se produce al presentarse una sequía, es la disminución del hato por ventas forzadas y sacrificio de ganado en mala condición física; ello representa una reducción significativa del inventario del ganado y descapitalización del sector. Así mismo, la viabilidad económica de la actividad productiva se ve disminuida. México exportó poco más de 1,3 millones de becerros a EUA, $85 \%$ becerros y $15 \%$ vaquillas. El estado de Sonora ocupó el primer lugar con $24,5 \%$ y Chihuahua, el segundo, con $23,2 \%$ (SNIIM, 2013).

El objetivo del presente estudio fue analizar la situación microeconómica de Unidades Representativas de Producción (URP) del sistema de producción vacabecerro en el estado de Chihuahua, México.

\section{MATERIALES Y MÉTODOS}

Los datos para el análisis se obtuvieron de enero a diciembre de 2010 en el estado de Chihuahua, localizado al norte de México y correspondieron al ciclo productivo 2009.

Se consideraron las cuatro principales regiones productoras de carne bovina: Chihuahua, Nuevo Casas Grandes, Cuauhtémoc e Hidalgo del Parral. Los criterios para elegir las regiones productoras fueron la superficie utilizada y el volumen producido de carne en canal. Así mismo, la Unidad Representativa de Producción (URP) elegida, representó en promedio el $30 \%$ de los productores de cada región.

A cada URP se le asignó un código de identificación, las dos primeras letras corresponden a la entidad federativa (Chihuahua), las siguientes al sistema de producción (bovinos carne) y el número a la escala de producción (número de vientres en producción): CHBC20, CHBC40, CHBC200 y CHBC500. Para el análisis se utilizó la unidad animal (UA), vaca de 450 $\mathrm{kg}$ con su cría o su equivalente en unidad animal (450 $\mathrm{kg}$ de peso vivo) como fuente de ingreso.

Otros indicadores de eficiencia técnica que permiten analizar la situación microeconómica de un sistema de producción de bovinos de carne son: relación vaca:toro, porcentaje de pariciones, mortalidad, coeficiente de agostadero (Cuadro 1). Según Hamilton (2006) un toro con fertilidad media puede servir hasta cuarenta vacas.

Los datos de costos e ingresos se obtuvieron a través de la técnica de panel (se define como la unidad representativa o simulada, que sin representar a un productor en particular, representa a los productores de la misma escala de producción y de la región), conformado por un grupo de productores representativo de cada región, sistema de producción, nivel tecnológico y escala de producción. Los grupos se integraron entre cinco y diez productores líderes, convocados y coordinados por un facilitador o persona que conoce a los productores. Durante el panel, se recabó información de ingresos y costos de producción del ciclo productivo 2009. La escala, sistema de producción, nivel tecnológico, precio de compra de insumos y venta de productos, fueron acordados y consensados por panelistas. 
Cuadro 1. Características de la Unidad Representativa de Producción (URP) en bovinos (vaca-becerro). Chihuahua, México. 2011.

\begin{tabular}{lcccc}
\hline URP & $\begin{array}{c}\text { Escala } \\
\text { (vientres) }\end{array}$ & $\begin{array}{c}\text { Superficie utilizada } \\
\text { (ha) }\end{array}$ & $\begin{array}{c}\text { Valor de los activos } \\
(\mathbf{1 0 0 0} \text { pesos })\end{array}$ & $\begin{array}{c}\text { Mercado del producto } \\
\text { final }\end{array}$ \\
\hline CHBC20 & 20 & 56 & 408 & Regional \\
CHBC40 & 40 & 170 & 1645 & Regional \\
CHBC200 & 200 & 2000 & 8321 & Exportación \\
CHBC500 & 500 & 10000 & 14194 & Exportación \\
\hline
\end{tabular}

Como indicadores de eficiencia microeconómica se consideró el capital neto, el ingreso neto, la relación beneficio-costo, el ingreso en efectivo, el costo total, la reserva de capital, el capital neto real y el valor presente neto; los cuales fueron obtenidos utilizando el programa econométrico MEXSIM, desarrollado por la Texas Extension and Education Foundation (TEEF), adscrita al Food and Agricultural Policy Center (AFPC) de Texas A\&M University (TAMU), el cual se utilizó en EE.UU. para analizar cambios en la Ley Agrícola (Farm Bill) antes de su implementación. El MEXSIM es un modelo de simulación de sistemas de producción tipo Monte Carlo, utilizado para analizar la viabilidad económica de URP agropecuarias en México. El modelo se programa en Excel y usa funciones de Simetar (Simulation and Econometric to Analyze Risk) para simular condiciones de riesgo e incertidumbre.

La situación financiera resume la eficiencia económica, liquidez y solvencia de la URP. Esta se consideró en buena situación financiera, cuando la probabilidad de reservas finales de efectivo negativas y de perder capital (patrimonio o capital neto real) fueron menores a $25 \%$; marginal cuando ambas probabilidades estuvieron entre 25 y $50 \%$ y pobre cuando fueron superiores a $50 \%$.

Las reservas en efectivo son el saldo al final del ciclo productivo, se obtiene al restar al efectivo final las reservas de efectivo inicial, más ingreso neto e intereses ganados sobre reservas, menos abonos a capital (préstamos), impuestos, gastos de manutención de la familia, y costo de reemplazo de la maquinaria. Así mismo, el capital o patrimonio neto nominal, resulta de restar la deuda total al total a todos los activos, incluyendo la tierra. Finalmente, el capital o patrimonio neto real (capitalización) es el cambio anualizado, en porcentaje, del patrimonio neto real; proyectado del 1 de enero de 2010 al 31 de diciembre de 2017.

Se utilizó un tipo de cambio de 13,5095 pesos por dólar promedio anual de México en 2009 para representar los valores monetarios.

\section{RESULTADOS Y DISCUSIÓN}

Las URP CHBC200 y CHBC500 fueron las principales productoras y exportadoras de becerros a los Estados Unidos, las de mayor inversión en infraestructura, genética, sanidad y manejo del hato. El sistema de producción vaca-becerro se caracterizó por utilizar grandes extensiones de tierra, genética del ganado acorde con características del medio ambiente, mercado de destino y baja inversión en activos productivos, los cuales se encontraron en su mayoría, depreciados. La superficie utilizada en USA para MTB500 (USA) es de 5665 hectáreas y TXRB500 de 8093 hectáreas (Sartwelle, 2006).

\section{Eficiencia técnica}

El peso de becerros destetados varió entre 130 y $200 \mathrm{~kg}$ en pie, las vacas de $450 \mathrm{~kg}$ y sementales de 600 a $700 \mathrm{~kg}$. El porcentaje promedio de pariciones (71\%) guarda una relación directa con la escala de producción; en las URP pequeñas fue $60 \%$ y en las grandes mayor a $80 \%$. Esta relación se explica por la calidad genética de animales, alimentación, relación vaca-toro, mortalidad y manejo de la URP. La relación vaca-toro fue baja en CHBC200 (8:1) y alta en CHBC40 (40:1). La mortalidad, promedio, para todas las URP fue de $2,3 \%$, por debajo del promedio nacional $(2,6 \%)$. La tasa de extracción en vacas fue $11 \%$ y nula en toros para tres URP a excepción de CHBC500 (18\%); 37\% de becerras 
nacidas en la URP se utilizó como reemplazo, más alta para las URP pequeñas que para grandes. Al considerar el coeficiente de agostadero máximo (SEMARNAT, 2010), que para el estado de Chihuahua es 20 ha/UA, todas las URP estuvieron por debajo de este promedio, a excepción de la de mayor escala (Cuadro 2).

\section{Costo de producción}

El costo anual por vientre fue diferente para todas las URP. El más bajo lo tuvieron los productores con 500 vientres y el más alto los de 40 vientres (diferencia de 57\%). El costo anual por vientre y por región fue \$ 214,66 dólares (CHBC20), \$ 249,82 (CHBC40), $\$ 159,15$ (CHBC200) y $\$ 125,54$ (CHBC500).

En todas las URP los costos en combustibles y alimento fueron de los más significativos. El consumo de combustibles se asoció a la distancia que tiene que recorrer un productor desde su hogar hasta la URP, de la superficie de la unidad de producción y compra de alimento al bajo coeficiente de agostadero, que en Chihuahua fue, en promedio, de 20 ha/UA. El mantenimiento se refiere al costo de infraestructura, maquinaria y equipo (Figura 1 ).

El costo promedio por kilogramo producido de carne en pie (vacas, toros de desecho y crías al destete) fue $\$ 0,91$ y $\$ 1,93 \pm 0,67$ dólares considerando

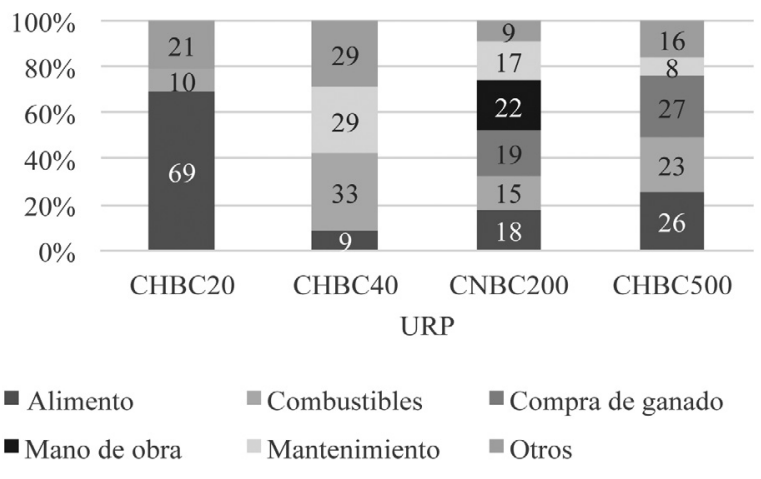

Figura 1. Integración del costo porcentual de producción, en Unidades Representativas de Producción (URP), de 20, 40, 300 y 500 bovinos de carne. Chihuahua (CH) y Nuevo Casas Grandes (CN), México. 2011.

solamente crías al destete. Por escala de producción, el costo más bajo correspondió a CHBC500 de 0,76 y \$ 0,99 dólares por kg y más alto en CHBC40 de 3,01 y 2,30 dólares, respectivamente. Por vientre, el promedio fue de 187,29 $\pm 55,57$ dólares; fue más alto en CHBC40 de 249,82 dólares y más bajo en CHBC500 de 125,54 dólares. Por hectárea, el promedio fue de 39,41 33,73 dólares; fue más alto en CHBC20 de 76,67 y más bajo en CHBC500 de 6,28 dólares.

Cuadro 2. Características de eficiencia técnica de las Unidades Representativas de Producción (URP) con 20, 40, 200 y 500 unidades de bovinos de carne en cuatro regiones de Chihuahua, México. 2011.

\begin{tabular}{llcccc}
\hline Variables productivas & & \multicolumn{3}{c}{ Unidades representativas de producción } \\
\cline { 3 - 6 } & & CHBC20 & CHBC40 & CHBC200 & CHBC500 \\
\hline Pariciones (\%) & 60 & 60 & 85 & 80 \\
Relación vaca-toro & Toros & 30 & 40 & 8 & 17 \\
Mortalidad (\%) & Vacas & 3 & 2 & 0 & 2 \\
& Becerros & 3 & 2 & 2 & 2 \\
Vientres desechados (\%) & Vacas & 12 & 10 & 10 & 0 \\
& Toros & 0 & 0 & 28 & 12 \\
Reemplazos nacidos (\%) & & 45 & 40 & 10 & 35 \\
Carga animal (ha/vientre) & & 2,8 & 4,3 & & 20 \\
\hline
\end{tabular}

CHBC20: Chihuahua Bovinos Carne 20 vientres; CHBC40: Chihuahua Bovinos Carne 40 vientres; CHBC200: Chihuahua Bovinos Carne 200 vientres; CHBC500: Chihuahua Bovinos Carne 500 vientres. 


\section{Ingreso}

El ingreso de todas las URP fue positivo y se derivó de la venta de crías, vacas de desecho, toros y transferencias del gobierno. En el caso de CHBC20, su principal fuente de ingreso fue la venta de leche y para las demás URP, la venta de becerros destetados (Figura 2).

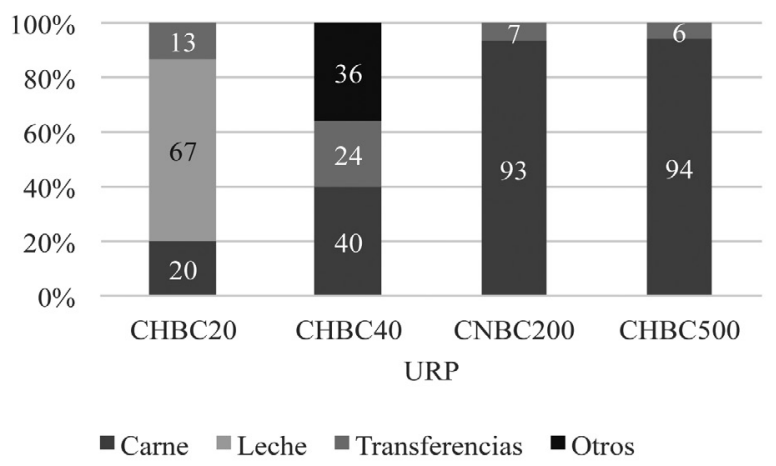

Figura 2. Composición porcentual del ingreso por Unidades Representativas de Producción (URP) con 20, 40, 200 y 500 unidades de bovinos de carne. Chihuahua, México. 2011.

El ingreso total promedio por vientre fue $\$ 359,08$ $\pm 174,72$ dólares; para CHBC20 de $\$ 618,08$, CHBC40 \$277,58, CHBC200 \$ 302,75 y CHBC500 \$ 237,91 dólares. Por hectárea, el ingreso total promedio fue \$ $39,41 \pm 33,73$ dólares; CHBC20 de \$76,67, CHBC40 de 58,78, CHBC200 de $\$ 15,91$ y CHBC500 \$ 6,28 dólares. Por becerro producido de $\$ 476,66 \pm 276,70$ dólares en promedio; para CHBC20 de $\$ 882,98$, CHBC40 de $\$ 370,11$, CHBC200 de $\$ 356,18$ y CHBC500 de $\$ 297,38$ dólares por cabeza. Finalmente, por kilogramo producido en pie de $\$ 1,41 \pm 1,51$ dólares.

Para un rancho de 500 vientres en California, Montana y Texas el ingreso bruto promedio por vientre fue de $\$ 555,26, \$ 577,63$ y 846,98 dólares respectivamente; en tanto que para uno de 200 vientres en Texas fue de \$754,95 dólares (Richardson et al., 2011).

A excepción de CHBC20 donde el ingreso neto de la actividad permitió cubrir solo $70 \%$ del gasto familiar del productor, para las demás URP cubre el $100 \%$. Para CHBC40 y CHBC500 representó 1,5 veces su gasto familiar y para CHBC200, 2,4 veces (Figura 3).

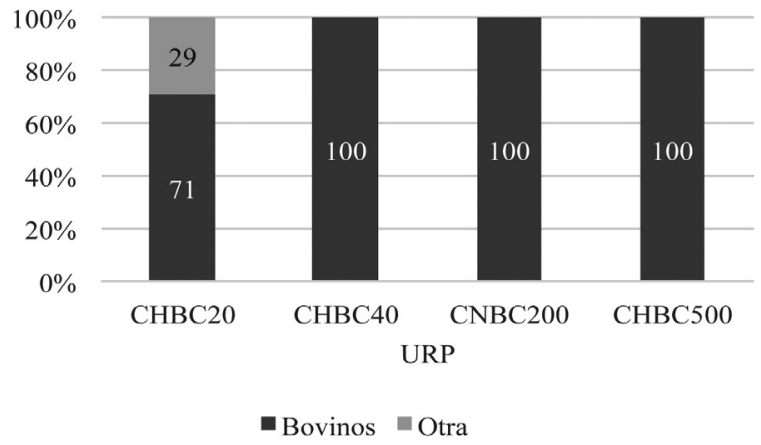

Figura 3. Ingresos netos de los productores por Unidades Representativas de Producción (URP) con 20, 40,200 y 500 unidades de bovinos de carne e indicadores de desempeño económico. Chihuahua, México. 2011.

Urs y Workman (1997) en Zimbabwe, encontraron que la mayor parte del ingreso de productores de ganado bovino provino de la actividad, mientras que la combinación con la fauna silvestre fue más rentable.

La inversión de las URP no tuvo una relación directa con el tamaño del hato y el mercado del producto final. En el caso de las URP grandes su principal mercado fue la exportación y las pequeñas, local y/o regional. La inversión promedio por vientre fue $\$ 2439,64$ dólares; para CHBC20 de $\$ 1635,89$, CHBC40 de $\$ 2973,83$, CHBC200 de $\$ 3040,82$ y CHBC500 de \$2108,00 dólares.

La relación beneficio-costo, fue de 1,36 para CHBC20, 1,25 para CHBC40, 1,78 para CHBC200 y 2,16 para CHBC500, lo que indicó que todas las URP fueron rentables y hubo una relación directa con la escala de producción. La tasa interna de retorno (TIR) fue de 4,2\% para CHBC20, 4,7\% para CHBC40, $8,16 \%$ para $\mathrm{CHBC} 200$ y $5 \%$ para CHBC500. Así mismo, la tasa de retorno sobre activos fue $12 \%$ para CHBC20, $1 \%$ para $\mathrm{CHBC} 40,5 \%$ para $\mathrm{CHBC} 200$ y $8,59 \%$ para CHBC500.

La situación económica de bovinos carne en el estado de Chihuahua fue buena para tres unidades de producción y moderada para una. Las URP con mayor número de vientres en producción tuvieron un margen de ganancia mayor, con base en una mayor inversión en activos productivos, alimentación, manejo y calidad genética del hato. Así mismo, la eficiencia económica fue menor cuando el productor asoció la producción pecuaria con la agrícola, debido a los altos costos de esta última. Uno de los aspectos importantes en la 
calidad de becerros, fue la introducción de animales, genéticamente desarrollados para la producción de carne; sin embargo, los productores de escalas de producción más grandes, hicieron uso de razas mejoradas en mayor proporción que los pequeños.

\section{LITERATURA CITADA}

Aksoy, A., M. Kulekci, y T. Erem. 2009. An econometric analysis for future of cattle. A case study in Erzurum, Turkey. Journal of Animal and Veterinary Advances 8 (2):348-349.

Banco Mundial. 2007. Informe sobre el desarrollo mundial 2008. Agricultura para el desarrollo. http://siteresources.worldbank.org/INTWDR2008/ Resources/2795087-1192111580172/FINAL_WDR-OVSpanish-text_9.26.07.pdf (Consultado 5 agosto 2011).

Chauvet, M. 1997. La ganadería mexicana frente al fin de siglo. Departamento de Sociología, Universidad Autónoma Metropolitana Azcapotzalco, México.

Delgado, C., C. Narrod, y M. Tiongco. 2008. Determinants and implications of the growing scale of livestock farms in four fast-growing developing countries. Research Report 157. IFPRI, Washington, D.C., USA.

FAO (Food and Agriculture Organization of the United Nations). 2009. El estado mundial de la agricultura y la alimentación: la ganadería, a examen. Viale delle Terme di Caracalla. 0013 Roma, Italia. http://www. fao.org/docrep/012/i0680s/i0680s00.htm (Consultado 11 marzo 2011).

FAO. 2013. Ganadería primaria. http://faostat3.fao.org/ faostat-gateway/go/to/download/Q/QL/E. (Consultado 25 nov. 2013)

Hamilton, T. 2006. Factsheet. Beef bull fertility. No. 89-087. Ween's printer for Ontario. Canadá.

Osuna, S. 2003. La problemática de la ganadería en México. En: IX Encuentro Nacional de Legisladores del sector Agropecuario de México. Nuestro Congreso. Órgano Informativo del Congreso del Estado de Sinaloa. p. 86-90.
Richardson, J., J. Outlaw, G. Knapek, J. Raulston, D. Herbst, H. Anderson, S. Bryant, y P. Zimmer. 2011. Representative farms economic outlook for the January 2011. FAPRI/AFPC Baseline, USA.

Rodríguez, C. 2008. Suplementación práctica de ganado bovino en pastoreo. Universidad Autónoma de Chihuahua. Facultad de Zootecnia y Ecología. Secretaría de extensión y Difusión. Manual técnico No. 8.

Sartwelle, J.D., J.L. Outlaw, y J.W. Richardson. 1986. Financial impacts of regional differences in beef cattle operations. Selected paper prepared for presentation at the Southern Agricultural Economics Association Annual Meetings, Orlando, Florida, USA.

SIAP (Sistema de información agropecuaria). 2013. Mercados nacionales e internacionales. http://www. siap.gob.mx/index.php?option=com_content $\& v i e w=a$ rticle\&id=26\&Itemid=333 (Consultado 26 sep. 2013).

SEMARNAT (Secretaría del Medio Ambiente y Recursos Naturales). 2010. Compendio de estadísticas ambientales. SAGARPA. Comité Técnico Consultivo de Coeficientes de Agostadero (Cotecoca). Junio 2009. http://aplicaciones.semarnat.gob.mx/estadisticas/ compendio2010/10.100.13.5_8080/ibi_apps/ WFServlet77fe.html (Consultado 20 enero 2011).

SNIIM (Sistema Nacional de Información e Integración de Mercados). 2013. Mercados del exterior. http://www. economia-sniim.gob.mx/nuevo (Consultado 6 sep. 2013).

Tinoco, R., D. Martínez, R. García, G. Hernández, y S. Mora. 2011. Aplicación de un sistema de demanda casi ideal (AIDS) a cortes de carnes de bovino, porcino, pollo, huevo y tortilla en el periodo de 1995-2008. Revista Mexicana de Ciencias Pecuarias (2)1:39-51.

Urs, P., y J. Workman. 1997. Comparative profitability of cattle and wildlife ranches in semi-arid Zimbabwe. Journal of Arid Enviroments 35:171-187.

United States Department of Agriculture. 2007. Census of agriculture. United States summary and state data. Volume 1. Geographic Area Series. Part 51. AC07-A-51. Updated December 2009. USA 